\title{
Effects of orthodontic experience on the reliability of the GOSLON Yardstick
}

\author{
Aslıhan Uzel ${ }^{1}$, Servet Doğan ${ }^{2}$ \\ ${ }^{1}$ Department of Orthodontics, Faculty of Dentistry, Çukurova University, Adana, Turkey \\ ${ }^{2}$ Department of Orthodontics, Faculty of Dentistry, Ege University, İzmir, Turkey \\ Email: asliuzel@cu.edu.tr
}

Received 3 July 2012; 6 August 2012; 16 August 2012

\begin{abstract}
Objective: The aim of this study was to investigate the importance of the clinical experience on the reliability of the GOSLON Yardstick. Design: Comparativemeasures study. Material: 3D images of the study models of children with complete unilateral cleft lip and palate (n: 119) at an average age of 9 years were included to the study. All subjects had their cleft lip and palate repaired in many hospitals by different surgeons, but no alveolar graft or any orthodontic treatment was performed. Method: Digital views of the models were used by OrthoModel software program. 3D images of the study models were scored by two groups of raters (Group I: 10 experienced orthodontists, Group II: 10 post-graduate orthodontic students) after careful perusal of published literature concerning GOSLON scoring and examining dental models samples. Main outcome measures: Statistics of intra- and inter-rater agreement. Results: Intra-rater agreement measurements of the two groups were high and high inter-rater agreement was also found between the two groups. Conclusion: Based on the results of this study clinical orthodontic experience does not affect significantly the reliability of the GOSLON Yardstick when all the raters trained before scoring.
\end{abstract}

Keywords: GOSLON Yardstick; Unilateral Cleft Palate; Three-Dimensional Images; Clinical Experience

\section{INTRODUCTION}

The comparison of dental arch relationship of children with cleft lip and palate (CLP) has become a common practice. Beside intercenter studies, a lot of single units from all over the world audit their treatment outcomes to assess performance for the evidence based review of practice and the GOSLON (Great Ormond Street London and Oslo, Norway) Yardstick has become the tool of choice [1-19]. The GOSLON Yardstick was developed in 1987 by Mars et al. to grade dental arch relationships into the five categories in the late mixed and/or early permanent dentition in children with unilateral cleft lip and palate (UCLP). The GOSLON categories range from 1 , suggesting an excellent treatment outcome, to 5, suggesting a very poor outcome [20]. According to the GOSLON scale, categories 1 and 2 have occlusions that require simple orthodontic treatments and category 3 needs complex orthodontic treatment. Individuals in the category 4 are at the limits of orthodontic treatment, and orthognathic surgery will generally be necessary; whereas, subjects in the category 5 require combined orthodontic-surgical therapy.

The anteroposterior relationship was considered to be the most important clinically; whereas, vertical and transverse relationships helped discriminate borderline cases [20].

Since its first publication, it has been frequently used worldwide in comparative and outcome assessment studies. It has been proven to be a valid, reliable and reproducible in many settings $[8,11,12,17,20]$. Although the GOSLON score is an efficient instrument when using by certified raters, the requirement of a substantial degree of professional judgment with regard to the possibility of orthodontic correction, have been suggested as a limitation of the Yardstick [21,22]. However, it has not published yet any study evaluating and proving that suggestion.

Thus, the aim of the present study was to investigate the reliability of the GOSLON Yardstick when used by inexperienced raters. The null hypothesis was "Orthodontic experience does not affect the reliability of the GOSLON Yardstick when all raters trained before scoring."

\section{MATERIALS AND METHOD}

3D images of the study models of children with complete unilateral cleft lip and palate (n: 119) at an average age of 9 years ( $\min 8 \mathrm{yrs}-\max 11 \mathrm{yrs})$ were included to the 
study. All subjects had their cleft lip and palate repaired in many hospitals by different surgeons, but no alveolar graft or any orthodontic treatment was performed.

The dental casts of all patients were scanned. Digital views of the model casts were used by a software program of digital orthodontic dental cast archiving system (OrthoModel V1.01, Istanbul, TURKEY) (Figure 1). The software program allows assessors to analyze the images in every aspect by manipulating the digital models on the computer. Two groups of assessors gathered for GOSLON scoring. Group I consisted of 10 experienced orthodontists with a minimum of 5 years experience in the treatment of cleft lip and palate patients. Group II consisted of 10 post-graduate orthodontic students. For the purpose of training published literature concerning GOSLON scoring was carefully perused and sample models were jointly examined by all the raters. After training period, 3D images of the 119 study models were projected in a conference room. The illustrations and description of GOSLON Yardstick given in the publication of Mars et al. (1987) was available for every assessor for inspection during scoring however, no conferring among assessors was allowed 20. Second examination was performed one month later by five assessors from each group. That time each rater assessed the images on a CD using his/her own computer.

The linear weighted kappa statistical test (computing a lower $95 \%$ confidence interval [CI]) was used to evaluate intra-and inter-rater reliability scores for the assessors.

\section{RESULTS}

Intra-rater agreement measurements of the two groups were high (weighted kappa coefficient: 0.89), and high inter-rater agreement was also found between the two groups (weighted kappa coefficient: 0.91). Weighted kappa statistics for inter-rater and intra-rater agreements are shown in Tables 1-4. The mean GOSLON score were 3.26 and 3.20 respectively for the Groups I and II and no statistical significance was found between two scoring.

\section{DISCUSSION}

The need for interdisciplinary treatment for the well being of patient with CLP is very well known [23-26]. However, there is a diversity of treatment protocols applied by different centers worldwide. Improvements in the treatment of cleft lip and palate require the determination of optimal treatment protocols via clinical trials to ensure that such protocols are being successfully applied. Thus, outcome assessment and reporting by cleft lip and palate units are gained more and more importance to assess the efficacy of treatment and whether changes are required [4,15,24,27-32]. Therefore, it is essential to have valid and reliable outcome measures to carry out all the studies mentioned above [15,20,33]. GOSLON Yardstick is a universally accepted tool for the comparison of dental arch relationship outcomes of children with CLP. In the Eurocleft studies, the GOSLON Yardstick

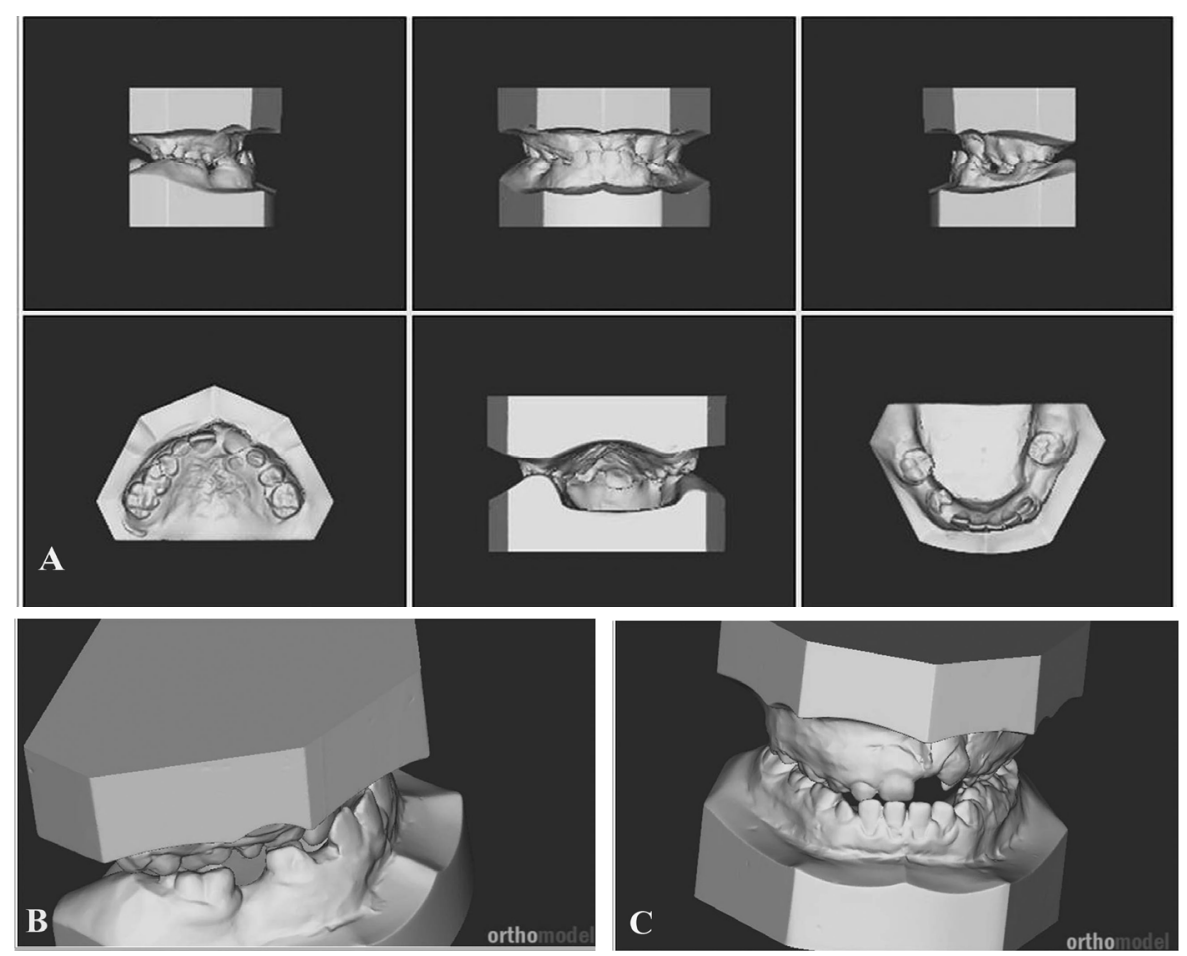

Figure 1. 3D images of dental casts (A): Standard six views, $(B, C)$ : Rotated views. 
Table 1. Inter-rater agreement for Group I (weighted Kappa statistics).

\begin{tabular}{cccccccccc}
\hline & $\mathbf{A 2}$ & $\mathbf{A 3}$ & $\mathbf{A 4}$ & $\mathbf{A 5}$ & $\mathbf{A 6}$ & $\mathbf{A 7}$ & $\mathbf{A 8}$ & $\mathbf{A 9}$ & $\mathbf{A 1 0}$ \\
\hline $\mathbf{A 1}$ & 0.99 & 0.99 & 1 & 0.99 & 0.99 & 1 & 0.99 & 0.98 & 0.99 \\
$\mathbf{A 2}$ & & 0.98 & 0.99 & 0.98 & 0.98 & 0.99 & 0.98 & 0.98 & 0.98 \\
$\mathbf{A 3}$ & & & 0.99 & 0.99 & 0.99 & 0.99 & 0.99 & 0.98 & 0.98 \\
A4 & & & & 0.99 & 0.99 & 1 & 0.99 & 0.98 & 0.99 \\
A5 & & & & & 0.99 & 0.99 & 0.99 & 0.98 & 0.98 \\
A6 & & & & & 0.99 & 0.99 & 0.99 & 0.98 \\
A7 & & & & & & & 0.99 & 0.98 & 0.99 \\
A8 & & & & & & & 0.98 & 0.98 \\
A9 & & & & & & & & 0.98 \\
\hline
\end{tabular}

Table 2. Inter-rater agreement for Group II (weighted Kappa statistics).

\begin{tabular}{lccccccccc}
\hline & B2 & B3 & B4 & B5 & B6 & B7 & B8 & B9 & B10 \\
\hline B1 & 0.75 & 0.67 & 0.76 & 0.66 & 0.81 & 0.77 & 0.78 & 0.80 & 0.77 \\
B2 & & 0.71 & 0.80 & 0.79 & 0.78 & 0.87 & 0.82 & 0.75 & 0.85 \\
B3 & & & 0.79 & 0.67 & 0.72 & 0.73 & 0.77 & 0.71 & 0.74 \\
B4 & & & & 0.74 & 0.86 & 0.84 & 0.85 & 0.81 & 0.81 \\
B5 & & & & & 0.75 & 0.77 & 0.81 & 0.74 & 0.83 \\
B6 & & & & & 0.82 & 0.92 & 0.95 & 0.78 \\
B7 & & & & & & 0.86 & 0.79 & 0.84 \\
B8 & & & & & & & 0.89 & 0.85 \\
B9 & & & & & & & & 0.77 \\
\hline
\end{tabular}

Table 3. Inter-rater agreement between groups (weighted Kappa statistics).

\begin{tabular}{cccc}
\hline & Kappa value & $\begin{array}{c}\text { Standart error } \\
\text { of Kappa }\end{array}$ & $\begin{array}{c}\mathbf{9 5 \%} \text { Confidence } \\
\text { Intervals }\end{array}$ \\
\hline Group I & & & \\
Versus & $0.83-0.95$ & 0.08 & $0.80-0.93$ \\
Group II & & & \\
\hline
\end{tabular}

Table 4. Intra-rater agreement (weighted Kappa statistics).

\begin{tabular}{cccc}
\hline Groups & Kappa value & $\begin{array}{c}\text { Standart error } \\
\text { of Kappa }\end{array}$ & $\begin{array}{c}\text { 95\% Confidence } \\
\text { Intervals }\end{array}$ \\
\hline I & $0.98-1$ & 0.08 & $0.82-0.9$ \\
II & $0.66-0.95$ & 0.08 & $0.73-0.83$ \\
\hline
\end{tabular}

was found to be a more sensitive tool in discriminating differences in outcomes of dentofacial growth in the participating centers than the cephalometric analysis of the same patients $[1,8]$. As it is used worldwide in different countries and centers, training of the investigators in scoring GOSLON models have been suggested for standardization of the ratings. Internet course with appropriate photographs of plaster casts of all different GOSLON types have been proposed as an economical alternative [22]. GOSLON was originally applied to dental cast. However, difficulties in traveling the plaster casts and possible damages lead investigators to use photographic methods. Nollet et al. [9] found that "photographs of dental casts provide a consistent, reproducible method for rating arch relationship in patients with UCLP and provide a reliable alternative to the application of the GOSLON Yardstick on dental casts". The validity and reliability of the intraoral photography method also proved to be good [34,35]. In a recent study Doğan et al. [36] showed that 3D images may provide a good alternative to the application of the Goslon Yardstick on dental casts and 2D images. Thus, in this study 3D images of dental casts were used and this allowed the examiners to rotate the images in various directions on the screen and to assess the overjet and overbite relationships more accurately than 2D images $[2,34,37]$.

Ten faculty member orthodontists and ten orthodontic students from different universities rated the 3D images for GOSLON scoring. The main reason of the meeting was to discuss the cleft care in Turkey and give a start to a study comparing dentoalveolar outcomes of patients with UCLP from different regions. Cleft patients are treated mostly in the university clinics in Turkey and orthodontic students get also involved in the orthodontic treatments of those patients.

Thus, it is important that students are able to evaluate treatment outcomes related to orthodontics. It is been suggested that subjectivity based on experience in GOSLON scoring might adversely affect the reliability of the Yardstick [21]. In this study we tended to assess whether orthodontic students can actively involve in the evaluation process of patients with UCLP and whether the GOSLON Yardstick can be reliably used by inexperienced raters. Sinko et al. [22] as a part of their study assessing dentoalveolar outcomes of Vienna sample, determined also intra and inter-rater agreement between several raters. They found no significant difference between ratings of an orthodontist and a dental student (0.82). A large weighted kappa value $(0.83-0.95)$ was found in our study which indicates a strong agreement between the scoring of the two groups. Intra-examiner agreement was larger for the Group I. However, kappa value was also acceptable for the Group II between two assessments with one month interval. Thus, it appears that personal training of the raters is more important than clinical orthodontic experience to reliably use the GOSLON Yardstick.

\section{CONCLUSION}

Based on the results of this study clinical orthodontic experience does not affect significantly the reliability of the GOSLON Yardstick when all raters trained before scoring.

\section{ACKNOWLEDGEMENTS}

Authors like to thank to all raters who participated in GOSLON scoring, 
from the Universities of Ankara, Atatürk, Başkent, Çukurova, Diyarbakır, Ege, Erzurum, İstanbul, Marmara, Kırıkkale, Yeditepe, Selçuk.

\section{REFERENCES}

[1] Mars, M., Asher-McDade, C., Brattsröm, V., Dahl, E., McWilliam, J., Mølsted, K., Plint, D.A., Prahl-Andersen, B., Semb, G., Shaw, W.C. and The, R.P.S. (1992) A sixcenter international study of treatment outcome in patients with complete cleft lip and palate: Part 3. Dental arch relationships. Cleft Palate-Craniofacial Journal, 29, 405-408.

doi:10.1597/1545-1569(1992)029<0405:ASCISO >2.3.C $\underline{\mathrm{O} ; 2}$

[2] Hathorn, I., Roberts-Harry, D. and Mars, M. (1996) The Goslon Yardstick applied to a consecutive series of patients with unilateral clefts of the lip and palate. Cleft Palate-Craniofacial Journal, 33, 494-496. doi:10.1597/1545-1569(1996)033<0494:TGYATA $>2.3 . \mathrm{C}$ $\underline{\mathrm{O} ; 2}$

[3] Morris, D.O., Roberts-Harry, D. and Mars, M. (2000) Dental arch relationships in Yorkshire children with unilateral cleft lip and palate. Cleft Palate-Craniofacial Journal, 37, 453-462.

doi:10.1597/1545-1569(2000)037<0453:DARIYC $>2.0 . C$ $\underline{\mathrm{O} ; 2}$

[4] Williams, A.C., Bearn, D., Mildinhall, S., Murphy, T., Sell, D., Shaw, W.C., Murray, J.J. and Sandy, J.R. (2001) Cleft lip and palate care in the United Kingdom-The Clinical Standards Advisory Group (CSAG) study. Part 2: Dentofacial outcomes and patient satisfaction. Cleft Palate-Craniofacial Journal, 38, 24-29. doi:10.1597/1545-1569(2001)038<0024:CLAPCI $>2.0 . C$ $\underline{\mathrm{O} ; 2}$

[5] Chan, T.C., Hayes, C., Shusterman, S., Mulliken, J.B. and Will, L.A. (2003) The effects of active infant orthopedics on occlusal relationships in unilateral complete cleft lip and palate. Cleft Palate-Craniofacial Journal, 40, 511517. doi:10.1597/1545-1569(2003)040<0511:TEOAIO $>2.0 . C$ $\underline{\mathrm{O} ; 2}$

[6] Johnston, C.D., Leonard, A.G., Burden, D.J. and McSherry, P.F. (2004) A comparison of craniofacial form in Northern Irish children with unilateral cleft lip and palate treated with different primary surgical techniques. Cleft Palate-Craniofacial Journal, 41, 42-46. doi:10.1597/03-027

[7] Kitagawa, T., Kohara, H., Sohmura, T., Takahashi, J., Tachimura, T., Wada, T. and Kogo, M. (2004) Dentoalveolar growth of patients with complete unilateral cleft lip and palate by early two-stage Furlow and push-back method: Preliminary results. Cleft Palate-Craniofacial Journal, 41, 519-525. doi:10.1597/03-020.1

[8] Molsted, K., Brattsrom, V., Prahl-Andersen, B., Shaw, W.C. and Semb, G. (2005) The Eurocleft study: Intercenter study of treatment outcome in patients with complete cleft lip and palate: Part 3: Dental arch relationships. Cleft Palate-Craniofacial Journal, 42, 78-82.

doi:10.1597/02-119.3.1
[9] Nollet, P.J., Katsaros, C., Van't Hof, M.A. and KuijpersJagtman, A.M. (2005) Treatment outcome in unilateral cleft lip and palate evaluated with the GOSLON Yardstick: A meta-analysis of 1236 patients. Plastic \& Reconstructive Surgery, 116, 1255-1262. doi:10.1097/01.prs.0000181652.84855.a3

[10] Lilja, J., Mars, M., Elander, A., Enocson, L., Hagberg, C., Worrell, E., Batra, P. and Friede, H. (2006) Analysis of dental arch relationships in Swedish unilateral cleft lip and palate subjects: 20-year longitudinal consecutive series treated with delayed hard palate closure. Cleft Palate-Craniofacial Journal, 43, 606-611. doi:10.1597/05-069

[11] Mars, M., Batra, P. and Worrell, E. (2006) Complete unilateral cleft lip and palate: Validity of the five-year index and the GOSLON Yardstick in predicting long-term dental arch relationships. Cleft Palate-Craniofacial Journal, 43, 557-562. doi:10.1597/05-074

[12] Susami, T., Ogihara, Y., Matsuzaki, M., Sakiyama, M., Takato, T., Shaw, W.C. and Semb, G. (2006) Assessment of dental arch relationships in Japanese patients with unilateral cleft lip and palate. Cleft Palate-Craniofacial Journal, 43, 96-102. doi:10.1597/04-117R.1

[13] Nollet, P.J.P.M., Kuijpers-Jagtman, A.M., Chatzigianni, A., Semb, G., Shaw, W.A., Bronkhorst, E.M. and Catsaros, C. (2007) Nasolabial appearance in unilateral cleft lip, alveolus and palate: A comparison with Eurocleft. Journal of Cranio-Maxillofacial Surgery, 35, 278-286. doi:10.1016/j.jcms.2007.04.011

[14] Fudalej, P., Hortis-Dzierzbicka, M., Dudkiewicz, Z. and Semb, G. (2009) Dental arch relationship in children with complete unilateral cleft lip and palate following Warsaw (one-stage repair) and Oslo Protocols. Cleft Palate-Craniofacial Journal, 46, 648-653. doi:10.1597/09-010.1

[15] Zreaqat, M., Hassan, R. and Halim, A.S. (2009) Dentoalveolar relationships of Malay children with unilateral cleft lip and palate. Cleft Palate-Craniofacial Journal, 46, 326-330. doi:10.1597/07-210.1

[16] De Ruiter, A., Van der Bilt, A., Meijer, G. and Koole, R. (2010) Orthodontic treatment results following grafting autologous mandibular bone to the alveolar cleft in patients with a complete unilateral cleft. Cleft Palate-Craniofacial Journal, 47, 35-42. doi:10.1597/08-095.1

[17] Hathaway, R.R., Daskalogiannakis, J., Mercado, A.M., Russell, K.A., Long, R.E., Cohen, M., Semb, G. and Shaw, W.C. (2011) The Americleft study: An inter-center study of treatment outcomes for patients with unilateral cleft lip and palate: Part 2. Dental arch relationships. Cleft Palate-Craniofacial Journal, 48, 244-251. doi:10.1597/09-181.1

[18] Long, R.E. Jr., Hathaway, R.R., Daskalogiannakis, J., Mercado, A.M., Russell, K.A., Cohen, M., Semb, G. and Shaw, W.C. (2011) The Americleft study: An inter-center Study of treatment outcomes for patients with unilateral cleft lip and palate: Part 1. Principles and study design. Cleft Palate-Craniofacial Journal, 48, 239-243. doi:10.1597/09-180.1

[19] Love, R., Walters, M.J., Southall, P., Singer, S. and Gillett, D.A. (2011) Dental arch relationship outcomes in 
children with complete unilateral cleft lip and palate, treated at Princess Margaret Hospital for Children, Perth, Western Australia. Cleft Palate-Craniofacial Journal, 49, 456-462.

[20] Mars, M., Plint, D.A., Houston, W.J., Bergland, O. and Semb, G. (1987) The GOSLON Yardstick: A new system of assessing dental arch relationships in children with unilateral clefts of the lip and palate. Cleft Palate-Craniofacial Journal, 24, 314-322.

[21] Mossey, P.A., Clark, J.D. and Gray, D. (2003) Preliminary investigation of a modified Huddart/Bodenham scoring system for assessment of maxillary arch constriction in unilateral cleft lip and palate subjects. European Journal of Orthodontics, 25, 251-257. doi:10.1093/ejo/25.3.251

[22] Sinko, K., Caacbay, E., Jagsch, R., Turhani, D., Baumann, A. and Mars, M. (2008) The GOSLON Yardstick in patients with unilateral cleft lip and palate: Review of a Vienna sample. Cleft Palate-Craniofacial Journal, 45, 8792. doi:10.1597/06-118.1

[23] American Cleft Palate-Craniofacial Association (2000) Parameters for evaluation and treatment of patients with cleft lip/palate or other craniofacial anomalies. http://www.acpa-cpf.org

[24] Human Genetics Programme (2002) Global strategies to reduce the health-care burden of craniofacial anomalies: Report of WHO meetings on international collaborative research on craniofacial anomalies. WHO, Geneva.

[25] Watson A.C.H., Sell, D.A. and Grunwell, P. (2004) Management of cleft lip and palate. Whurr Publishers, London, Philadelphia.

[26] Berkowitz, S. (2006) Cleft lip and palate. 2nd Edition, Springer-Verlag Berlin Heidelberg, Berlin. doi:10.1007/3-540-30020-1

[27] Spriestersbach, D.C., Dickson, D.R., Fraser, F.C., Horowitz, S.L., McWilliams, B.J., Paradise, J.L. and Randal, P. (1973) Clinical research in cleft lip and cleft palate: The state of the art. The Cleft Palate Journal, 10, 113-165.

[28] Shaw, W.C., Asher-McDade, C., Brattstrom, V., Dahl, E., McWilliam, J., Molsted, K., Plint, D.A., Prahl-Andersen, B., Semb, G. and The, R.P.S. (1992) A six-center international study of treatment outcome in patients with clefts of the lip and palate: Part 1. Principles and study design. Cleft Palate-Craniofacial Journal, 29, 393-397. doi:10.1597/1545-1569(1992)029<0393:ASCISO >2.3.C $\underline{\mathrm{O} ; 2}$

[29] Shaw, W.C., Dahl, E., Asher-McDade, C., Brattstrom, V., Mars, M., McWilliam, J., Mølsted, K., Plint, D.A., Prahl-Andersen, B., Roberts, C., et al. (1992b) A six- center international study of treatment outcome in patients with clefts of the lip and palate: Part 5. General discussion and conclusions. Cleft Palate-Craniofacial Journal, 29, 413-418.

doi:10.1597/1545-1569(1992)029<0413:ASCISO >2.3.C $\underline{\mathrm{O} ; 2}$

[30] Long, R.E. Jr., Semb, G. and Shaw, W.C. (2000) Orthodontic treatment of the patient with complete clefts of the lip, alveolus and palate: Lessons of the past 60 years. Cleft Palate-Craniofacial Journal, 37, 533. doi:10.1597/1545-1569(2000)037<0533:OTOTPW $>2.0$. $\mathrm{CO} ; 2$

[31] Bearn, D., Mildinhall, S., Murphy, T., Murray, J.J., Sell, D., Shaw, W.C., Williams, A.C. and Sandy, J.R. (2001) Cleft lip and palate care in the United Kingdom-The Clinical Standards Advisory Group (CSAG) study. Part 4: Outcome comparisons, training, and conclusions. Cleft Palate-Craniofacial Journal, 38, 38-43. doi:10.1597/1545-1569(2001)038<0038:CLAPCI $>2.0 . C$ $\underline{\mathrm{O} ; 2}$

[32] Shaw, W.C., Brattstrom, V., Molsted, K., Prahl-Andersen, B., Roberts, C.T. and Semb, G. (2005) The Eurocleft study: Intercenter study of treatment outcome in patients with complete cleft lip and palate. Part 5: Discussion and conclusions. Cleft Palate-Craniofacial Journal, 42, 93-98. doi:10.1597/02-119.5.1

[33] Ozawa, T.O., Shaw, W.C., Katsaros, C., Kuijpers-Jagtman, A.M., Hagberg, C., Rønning, E. and Semb, G. (2011) A new yardstick for rating dental arch relationship in patients with complete bilateral cleft lip and palate. Cleft Palate-Craniofacial Journal, 48, 167-172. doi:10.1597/09-122

[34] Liao, Y.-F., Huang, C.-S. and Lin, I.-F. (2009) Intraoral photographs for rating dental arch relationships in unilateral cleft lip and palate. Cleft Palate-Craniofacial Journal, 46, 415-419. doi:10.1597/07-220.1

[35] Liao, Y.-F. and Lin, I.-F. (2009) Dental arch relationships after two-flap palatoplasty in Taiwanese patients with unilateral cleft lip and palate. International Journal of Oral \& Maxillofacial Surgery, 38, 1133-1136. doi:10.1016/j.ijom.2009.06.022

[36] Doğan, S., Olmez, S. and Semb, G. (2012) Comparative assessment of dental arch relationships using GOSLON Yardstick in UCLP patients using dental casts, 2D photos and 3D images. Cleft Palate-Craniofacial Journal, 49, 347-351.

[37] Garino, F. and Garino, G.B. (2002) Comparison of dental arch measurements between stone and digital casts. World Journal of Orthodontics, 3, 250-254. 